\title{
Tratamento de hérnia umbilical em bovinos ${ }^{1}$
}

\author{
Luiz Antônio Franco da Silva²*, Duvaldo Eurides, Luiz Augusto de Souza ${ }^{4}$, \\ Benito Juarez Nunes Alves de Oliveira ${ }^{4}$, Jalily Bady Helou ${ }^{5}$, Angela Moni Fonseca ${ }^{6}$, \\ Leonardo Lamaro Cardoso ${ }^{6}$, Sabrina Lucas Ribeiro de Freitas ${ }^{5}$
}

\section{RESUMO}

Bovinos jovens são frequentemente acometidos por hérnias umbilicais. Neste trabalho, avaliou-se a ocorrência e estabeleceu-se o tratamento cirúrgico para hérnias umbilicais em bovinos jovens, empregando 78 bezerros distribuídos em seis grupos. Em GI e GIV, na laparorrafia, utilizou-se sutura jaquetão; em GII e GV, jaquetão modificado e, em GIII e GVI, sutura separada simples com pontos de relaxamento. Em GI, GII e GIII utilizou-se fio de algodão e, em GIV, GV e GVI, de náilon. Dados não paramétricos, envolvendo a recuperação dos animais e sua independência ou associações com o padrão de sutura foram analisados pelo teste do Qui-quadrado e Exato de Fisher, fazendo-se uso do programa computacional Statistical Analysis System, a 5\% ( $\mathrm{p} \leq 0,05)$. Os demais resultados foram analisados descritivamente. A ocorrência de hérnia umbilical em bovinos foi expressiva e a herniorrafia, empregando-se fio de algodão, apresentou maiores complicações pós-operatórias; porém a maioria dos animais recuperou-se. O fio e o padrão de sutura, manejo pós-operatório, resposta individual e o tamanho do anel herniário influenciam na reabilitação dos animais e na redução das complicações pós-operatórias.

Palavras-chave: bezerro, cirurgia, hérnia, terapêutica, umbigo.

\section{ABSTRACT}

\section{Treatment of umbilical hernia in cattle}

Young cattle are commonly affected by umbilical hernias. Were evaluated the occurrence and surgical treatment for umbilical hernias in young cattle using 78 calves separated in six groups. Overlaying suture was used for laparorraphy in groups GI and GIV; modified overlaying in groups GII and GV; and simple separated suture with relaxing stitches in groups GIII and GVI. The material used for suture was cotton in groups GI, GII and GIII and nylon in GIV, GV and GVI. Non parametric data of animal recovery and independence or association between suture pattern were evaluated by $\mathrm{X}^{2}$ and Fisher Exact tests, using the software Statistical Analysis System with significance of 5\% (p $\leq 0.05)$. Other results were evaluated by descriptive statistics. The results showed high occurrence of bovine umbilical hernias and herniorraphy with cotton stitches had more postoperative complications, however most animals recovered after surgery. The thread and suture type, postoperative management, individual response and the size of hernia ring influence the recovery of the animal and reduction of postoperative complications.

Key words: calf, surgery, hernia, therapeutic, navel.

\footnotetext{
Recebido para publicação em 18/06/2010 e aprovado em 19/12/2011

${ }^{1}$ Fonte de Financiamento: CNPq, EV/UFG, EV/UFU e Iniciativa privada

${ }^{2}$ Médico Veterinário, Doutor. Universidade Federal de Goiás, Estrada Goiânia Nova Veneza Km 0, Campus II, Setor Samambaia, Caixa Postal 131, 74001-970, Goiânia, Goiás, Brasil. lafranco@vet.ufg.br (*Autor para correspondência)

Médico Veterinário, Doutor. Universidade Federal de Uberlândia, Avenida Pará, 1720, Campus Umuarama, 38400-902, Uberlândia, Minas Gerais, Brasil. duvaldo@ufu.br

${ }^{4}$ Médico Veterinário, Mestre. Universidade Federal de Goiás, Estrada Goiânia Nova Veneza Km 0, Campus II, Setor Samambaia, Caixa Postal 131, 74001-970, Goiânia, Goiás, Brasil. souza_vet@yahoo.com.br; benito@ortoneurovet.com

${ }_{5}^{5}$ Graduando em Medicina Veterinária. Universidade Federal de Goiás, Estrada Goiânia Nova Veneza Km 0, Campus II, Setor Samambaia, Caixa Postal 131, 74001-970, Goiânia, Goiás, Brasil. badyhelou@hotmail.com; sabrina_medvet@hotmail.com

${ }^{6}$ Médico Veterinário. Universidade Federal de Goiás, Estrada Goiânia Nova Veneza Km 0, Campus II, Setor Samambaia, Caixa Postal 131, 74001-970, Goiânia, Goiás, Brasil. angela_moni@hotmail.com; leolamaro@hotmail.com
} 


\section{INTRODUÇÃO}

Os bovinos jovens são frequentemente acometidos por enfermidades umbilicais, sendo as onfalites, onfaloflebites, miíases, fibrose e hérnias as de maior ocorrência (Silva et al., 2001; Erdoðan et al., 2009). A hérnia é uma protrusão do conteúdo de uma cavidade do corpo, através de abertura congênita ou adquirida, podendo ser classificada conforme a estrutura, alteração funcional, conteúdo e anatomia (Figueirêdo, 1999; Citek et al., 2009; Sutradhar et al., 2009), com ocorrência em todas as raças (Bahr \& Distl, 2005). Segundo Citek et al. (2009), os bovinos portadores dessa enfermidade não devem ser utilizados como reprodutores e, para Steenholdt \& Hernandez (2004), a hérnia pode estar associada a onfalopatias e manejo inadequado. Partos gemelares e antecipados são fatores de risco para ocorrência de hérnia umbilical em bezerros (Herrmann et al., 2001).

As hérnias umbilicais resultam em perda de peso, interferem no desenvolvimento dos animais, predispõem a infecções sistêmicas e podem desencadear o óbito, além de acarretarem desvalorização do animal e grandes prejuízos econômicos ao criatório (Rabelo et. al., 2005; Peiró et $a l .$, 2009). Os casos não tratados podem evoluir para aderências entre as estruturas contidas no saco herniário e peritônio (Rings, 1995). Conforme descrito por Silva et al. (2001) e Baird (2008), o conteúdo herniário com possível aderência do saco está composto na maioria dos casos de alças intestinais, peritônio e,ou, abomaso, sendo importante ao cirurgião diferenciar qual estrutura está presente. Segundo Silva et al. (2001), esta modalidade de hérnia pode estar relacionada com traumatismos como coices, pisadas de animais e transporte inadequado do bezerro. Figuerêdo (1999) citou que as hérnias umbilicais podem estar associadas a outras alterações patológicas que as tornam mais complicadas.

Para Figuerêdo (1999), o diagnóstico é fundamentado no exame semiológico, com o bovino em posição quadrupedal, pois tal conduta fornece melhores condições para as manobras de palpação e inspeção. As manifestações de dor, como inquietação, tentativa de lambedura e de coçar com os pés, estão associadas a aderências e suas complicações. Podem ser observadas, ainda, inapetência, febre, peritonite, obstrução, hemorragia abomaso-intestinal, falência orgânica e morte, nos casos severos. Para Figueirêdo (1999) e Baird (2008), a palpação requer suavidade, mas ao mesmo tempo deve ser firme, fornecendo ao examinador as características do conteúdo, presença de aderências, redução total ou parcial do saco herniário para a cavidade abdominal, identificação do tamanho do anel herniário e da presença de constituintes do cordão umbilical. Silva et al. (1999) e Silva et al. (2006) recomendam reduzir o conteúdo herniário e reconstituir o defeito na parede abdominal, para minimizar os prejuízos que as hérnias umbilicais podem acarretar.

De acordo com Turner \& Mclwraith (2002), quando as hérnias umbilicais nos bovinos não regridem espontaneamente, a intervenção cirúrgica é o melhor tratamento. Segundo Paulo et al. (2005), na eleição do procedimento cirúrgico reconstrutivo da parede abdominal deve-se considerar que, em geral, há perda dos diferentes planos anatômicos que a constituem. Portanto, a função de suporte perdida deve ser restabelecida e, sempre que possível, a pele deve ser suficiente para a cobertura total dos planos restaurados. Na herniorrafia, várias técnicas de sutura têm sido utilizadas, com resultados variáveis (Lazzeri, 1994; Silva et al., 1999; Sobayil \& Ahmed, 2007; Sutradhar et al., 2009). Portanto, não há dúvidas de que a hérnia umbilical constitua uma onfalopatia de grande significância no cenário da pecuária, comprometendo o desenvolvimento do bezerro, o valor comercial do animal e, consequentemente, acarretando prejuízos econômicos ao produtor. Sendo assim, apesar de esse assunto ser comentado por diversos autores (Lazzeri, 1994; Figueirêdo, 1999; Silva et al., 1999; Rabelo et al., 2005; Baird, 2008; Peiró et al., 2009; Sutradhar et al., 2009), ainda existem questionamentos sobre o tratamento e as principais complicações pós-operatórias, especialmente em propriedades rurais brasileiras.

Como muitas informações sobre o tema são provenientes de estudos realizados no exterior, em países cujas condições climáticas, valor do animal e recursos financeiros para o tratamento da enfermidade não são condizentes com a realidade dos criatórios nacionais, erros de conduta podem limitar a adoção de medidas preventivas e curativas mais adequadas, comprometendo a rentabilidade do criatório e tornando-o menos eficiente e econômico.

O objetivo deste trabalho foi avaliar a ocorrência, o tratamento cirúrgico e as complicações pós-operatórias das hérnias umbilicais em bovinos jovens, em algumas propriedades rurais dos Estados de Goiás, Distrito Federal e Minas Gerais, empregando-se casos atendidos entre os anos de 1989 a 2009.

\section{MATERIAL E MÉTODOS}

O estudo foi desenvolvido em propriedades rurais dos Estados de Goiás, Distrito Federal e Minas Gerais, entre 1989 e 2009, utilizando-se 78 bovinos jovens com hérnia umbilical, de um total de 24.860 animais com idade inferior a um ano, distribuídos entre zebuínos, taurinos e mestiços. Independentemente do sexo e da raça, primeiramente foi realizada a confirmação do diagnóstico, sendo, em seguida, os bovinos alocados em seis grupos de 13 animais cada, de acordo com o protocolo de tratamento estabelecido (GI, GII, GIII, GIV, GV e GVI). A cada 
atendimento foi confeccionada uma ficha com informações como raça, idade, sexo e número de animais na mesma faixa etária.

Todos os atendimentos foram realizados durante aulas práticas de Clínica Cirúrgica de grandes animais, ministradas por professores das Escolas de Veterinária das Universidades Federais de Goiás-GO e Uberlândia-MG, e execução de projetos de Extensão, desenvolvidos em diferentes propriedades rurais. O protocolo de tratamento adotado foi o cirúrgico, com abertura completa do saco herniário. O pré-operatório incluiu jejum completo de 12 horas, tranquilização com 0,1 a $0,2 \mathrm{mg} / \mathrm{kg}$ de Cloridrato de xilazina (Calmiun ${ }^{\circledR}$, Agener União Química, EmbuGuaçu, SP), quando se fez necessário, bem como contenção em decúbito dorsal com os membros locomotores estendidos. Realizou-se tricotomia ampla, higienização com água e sabão e antisepsia da região umbilical com iodopovidona (Iodopovidona ${ }^{\circledR}$, Farmogral, Brasília, DF). O bloqueio anestésico foi realizado ao redor do anel herniário e na linha de incisão com doses de 7 a 10 mg/kg de cloridrato de lidocaína (Dorfin ${ }^{\circledR}$, Hertape Calier Saúde Animal, Juatuba, MG).

Nos animais pertencentes aos grupos GI e GII, a laparorrafia foi praticada com padrão de sutura jaquetão, convencional e modificada, respectivamente. Para proceder à transformação desse padrão de sutura, empregouse uma das extremidades do fio e aplicou-se um ponto de reforço na fáscia abdominal externa, resultando em superposição tripla das fáscias. Nos animais alocados no grupo GIII, foi utilizado padrão de sutura separada simples, alternando-se com pontos de relaxamento. $\mathrm{O}$ fio de sutura utilizado, em todas as situações, foi algodão número 000. Já nos bovinos dos grupos GIV, GV e GVI, utilizou-se o fio de náilon número 0,50 , preparado artesanalmente, e o padrão de sutura obedeceu à mesma sequência estabelecida para os animais alocados nos três primeiros grupos: jaquetão convencional, jaquetão modificado e pontos separados, alternados com pontos de relaxamento (Tabela 1 e Figura 1). O espaço morto foi reduzido, empregando-se categute 0 (Med Line ${ }^{\circledR}$, Produtos Médicos Hospitalares e Laboratoriais LTDA, Goiânia, GO), em padrão Cushing. Na dermorrafia, de acordo com o grupo ao qual pertencia o animal, empregou-se o mesmo fio utilizado na laparorrafia: algodão ou náilon.

No pós-operatório, indicou-se aplicação tópica, na ferida cirúrgica e ao redor da lesão, de iodopovidona e matabicheira (Mata Bicheira Vetbrands ${ }^{\circledR}$, Vetbrands Saúde Animal, Jacareí, SP), diariamente. A antibioticoterapia parenteral constou da associação de benzilpenicilina procaína e sulfato de dihidroestreptomicina (Pentakel ${ }^{\circledR}$, Vetbrands, Jacareí, SP), na dose de $30.000 \mathrm{UI} / \mathrm{kg}$, por via intramuscular, a cada 48 horas, perfazendo cinco aplicações, e de flunixim meglumine (Banamine ${ }^{\circledR}$, ScheringPlough, São Paulo, SP), na dose de 1,1mg/kg, por três dias consecutivos. Recomendou-se a remoção dos pontos de pele no $12^{\circ}$ dia após o procedimento cirúrgico.

A evolução clínica e eventuais complicações pós-operatórias foram acompanhadas por médicos veterinários que assistiam às propriedades ou por um membro da equipe, por um período aproximado de 60 dias. Foram avaliadas possíveis complicações como edema, deiscência da ferida, miíases, abscesso, fibrose, recrudescimento da hérnia, evisceração e óbito. Paralelamente, identificaramse as principais causas das complicações, especialmente relacionadas com os fios empregados, padrões de sutura e manejo pós-operatório. Nesse caso, procurou-se analisar a estrutura física dos criatórios, a alimentação durante a fase de recuperação, a antibioticoterapia, as sujidades dos ambientes, os curativos e a mão de obra.

Dados não paramétricos, envolvendo a recuperação dos animais, empregando-se fio de algodão (GI, GIII, GV) e náilon (GII, GIV, GVI), foram analisados pelo teste do Qui-quadrado e Exato de Fisher. O mesmo teste foi empregado na análise dos resultados, envolvendo a independência ou associações com o padrão de sutura jaquetão convencional (GI e GIV), jaquetão modificado (GII e GV) e ponto separado simples com ponto de relaxamento (GIII e GVI). Para comparação entre os grupos, foi utilizado o teste de Qui-quadrado. Todas as análises foram realizadas, fazendo-se uso do programa computacional Statistical Analysis System (SAS, 2002), a 5\% ( $\mathrm{p} \leq 0.05)$ de significância ,conforme recomendação de Sampaio (2007). Os demais resultados foram analisados descritivamente.

Tabela 1. Distribuição dos bovinos em grupos de acordo com o fio e o padrão de sutura utilizado no tratamento cirúrgico de hérnia umbilical em bovinos jovens, em propriedades rurais do Estado de Goiás, Distrito Federal e Minas Gerais, entre 1989 e 2009

\begin{tabular}{lcl}
\hline Grupo & Fio de sutura & Padrão de sutura empregado na laparorrafia \\
\hline I & Algodão & Jaquetão convencional \\
II & Algodão & Jaquetão modificado \\
III & Algodão & Separado simples, alternando com pontos de relaxamento \\
IV & Náilon & Jaquetão convencional \\
V & Náilon & Jaquetão modificado \\
VI & Náilon & Separado simples, alternando com pontos de relaxamento \\
\hline
\end{tabular}



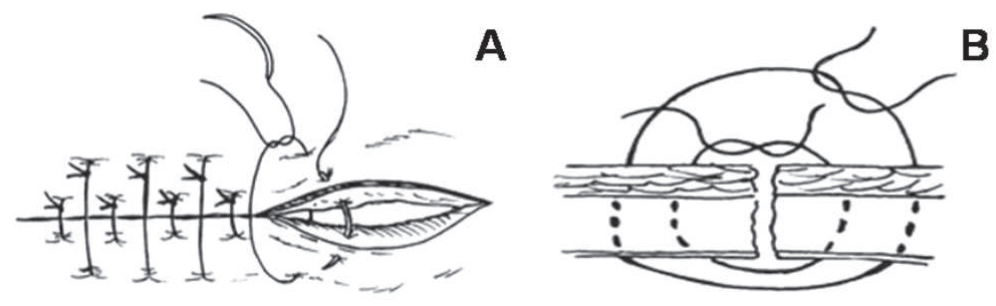

B
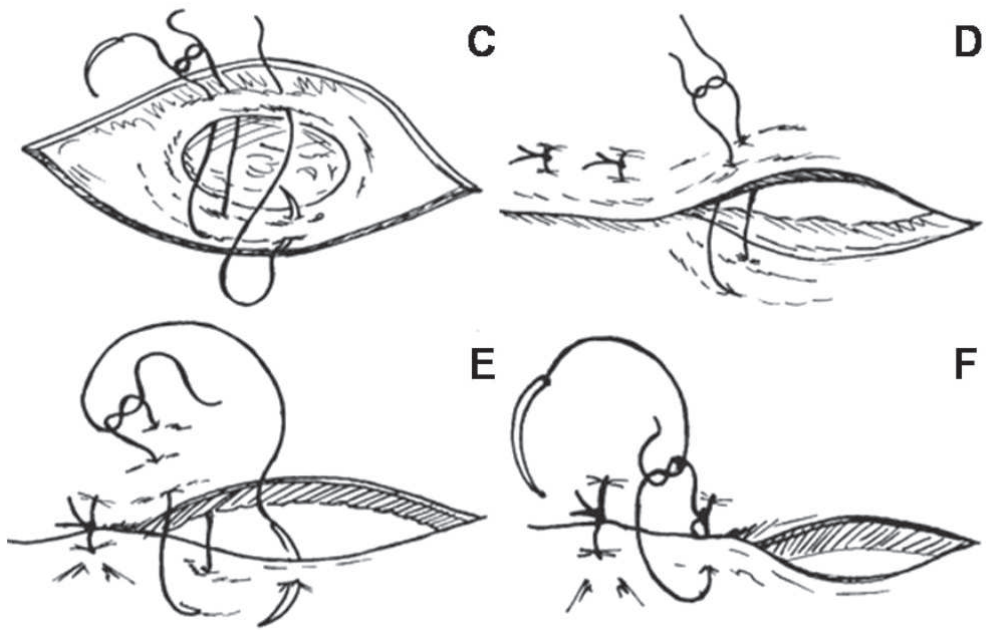

E

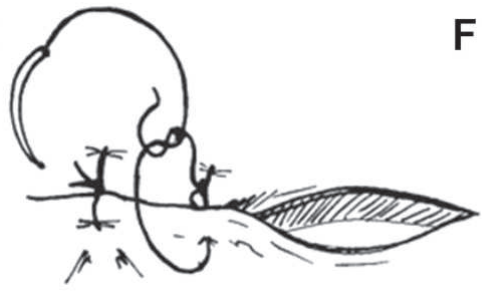

Figura 1. Padrões de suturas empregadas na herniorrafia umbilical em bovinos. A e B- Ponto simples separado, alternando com pontos de relaxamento; C e D- Jaquetão convencional; E e F- Jaquetão com ponto de reforço.

\section{RESULTADOS E DISCUSSÃO}

Do total de 24.860 animais, com idade entre sete e 240 dias, distribuídos entre raças de bovinos de aptidão para leite, corte e mista (Bos taurus X Bos indicus), em 78 $(0,31 \%)$ foi diagnosticada hérnia umbilical. Este número de animais comprometidos encontra-se dentro do intervalo estabelecido por Dias Filho et al. (2001) e Bahr \& Distl (2005). Conforme recomendaram Figueirêdo (1999) e Baird (2008), o diagnóstico foi obtido após exame clínico, não havendo a necessidade de se realizar a abdominocentese sugerida por Peiró et al. (2009), exame radiográfico apontado por Rings (1995), ou ultrassonográfico, recomendado por Steiner \& Lejeune (2009), os quais ressaltaram que o diagnóstico específico de transtorno das enfermidades umbilicais é importante, pois o tratamento, prognóstico e os custos dependem da extensão da doença e das estruturas envolvidas.

A insinuação de órgãos e estruturas da cavidade abdominal através do anel umbilical, bem como o omento maior, porção do intestino e a presença do anel inguinal foram achados importantes na confirmação do diagnóstico (Baird, 2008). Para Silva et al. (2001) e Andrews et al. (2008), a hérnia umbilical surge, geralmente, quando a linha alba não se fecha adequadamente ao redor da conexão abdominal do funículo umbilical. Peiró et al. (2009) apontaram que os achados laboratoriais são importantes nos animais com hérnia, pois ocorre aumento na proteína total do líquido peritoneal, na concentração de fibrino- gênio, linfócitos, aspartato aminotransferase sérica (AST), lactato desidrogenase aspartato $(\mathrm{LDH})$ e creatina quinase (CK), exames não realizados nos animais do presente estudo.

Nos casos mais simples e que não apresentavam sinais de aderências, o anel herniário variou de um a dez centímetros de diâmetro; o conteúdo era macio, redutível e indolor. Conforme verificado também neste estudo, Silva et al. (2001) e Andrews et al. (2008) relataram que pequenas hérnias com diâmetro menor que quatro centímetros geralmente fecham, espontaneamente, aos três ou quatro meses de idade, porém as hérnias umbilicais grandes ou estranguladas exigem correção cirúrgica, em especial quando estão associadas a infecções intra-abdominais, variando quanto ao índice de recuperação cirúrgica (Tabela 2).

Em relação ao tipo de fio empregado na sutura da parede abdominal, não houve diferença significativa $(\mathrm{p}=$ 0,055 ) entre os grupos submetidos ao tratamento com fio de algodão ou de náilon (Tabela 2). De forma semelhante à encontrada neste estudo, Sobayil \& Ahmed (2007) constataram que o material de sutura não influenciou no tratamento cirúrgico, embora os autores tenham utilizado caprinos, nas avaliações realizadas. Mas, ao contrário dos achados deste estudo, Sylvestre et al. (2002) afirmaram que os materiais de sutura interferem na cicatrização por meio da interação com o tecido. Sutradhar et al. (2009) recomendaram utilizar, na herniorrafia de bezerros mais 
velhos, material não absorvível, pois o material aumenta a proteção da área da ferida cirúrgica.

Mesmo não havendo diferença estatística, quando se utilizou o fio de algodão, a taxa de recuperação foi de $87,1 \%$ dos bovinos dos grupos GI, GII e GIII, enquanto, nos animais que constituíram os grupos GIV, GV e GVI, empregando-se o fio de náilon, apenas $69,2 \%$ se recuperaram. Sobre esses resultados pode-se argumentar que, possivelmente, o fato de o fio de algodão apresentar textura macia e pouco cortante não esgarçou a fáscia abdominal externa, ainda que a sutura tenha exercido tensão sobre os tecidos. Mas, ao contrário do efeito dessas características apresentadas pelo fio de algodão, o fio de náilon, especialmente nas suturas que resultaram em aumento de tensão, desencadeou dilaceração da fáscia, fato que pode ter contribuído para o recrudescimento do processo. Essa hipótese pode ser embasada nos resultados de Cuffari (1997) e Lazzeri (1999), os quais afirmaram que o fio de algodão tem maior capilaridade, baixa resistência tênsil, melhor absorção de fluido e alta reação tecidual, desencadeando, assim, maior fibrose no local. Valendose dessas afirmações, em tese, podem-se justificar os bons resultados obtidos na herniorrafia. Mas, por sua natureza física e química, este tipo de fio poderá desencadear maior número de complicações, além de predispor à possível contaminação.

Outro aspecto marcante do fio de náilon está relacionado com a dificuldade de confeccionar os nós, durante o trans-operatório, conferindo menor segurança à sutura. Portanto, dependendo da tração exercida sobre o fio, os nós não se mantinham amarrados, situação evidenciada por Lazzeri (1999), Rabelo (2003) e Fossum (2008). Essa intercorrência foi abolida, aplicando-se vários nós sobrepostos, prevenindo-se o deslizamento e consequente deiscência da ferida. Turner \& Mcilwraith (2002) afirma- ram que o material de sutura na herniorrafia é uma questão de escolha, contudo, enfatizaram suas preferências pela sutura absorvível sintética, como as suturas à base de ácido poliglicólico ou polyglactin. Porém, segundo estes autores, caso a hérnia seja recidiva, recomenda-se o uso de suturas inabsorvíveis sintéticas, empregando-se fios trançados sob condição asséptica. Na Tabela 3, apresentam-se os resultados do tratamento, considerando os padrões de sutura em relação aos fios empregados.

Associando-se os valores das três técnicas de sutura e avaliando-se o provável efeito desse parâmetro sobre a recuperação dos animais, independentemente do fio empregado, nota-se que houve diferença estatística entre os grupos, ao se compararem os resultados do jaquetão modificado e os do ponto separado simples, alternado com ponto de relaxamento $(\mathrm{p}=0,049)$. Entretanto, não se verificou diferença entre jaquetão convencional e jaquetão modificado $(\mathrm{p}=0,212)$ e, também, ponto separado simples $(p=0,442)$ (Tabela 3$)$. Porém, os números indicaram que ponto simples separado, alternado com ponto de relaxamento resultou em maior taxa de recuperação, sugerindo que esta técnica foi a mais eficaz, concordando com os achados de Silva et al. (2000). Para os autores, o insucesso das herniorrafias e os altos índices de recidivas podem ser atribuídos, em parte, à resposta individual dos animais, às falhas no pós-operatório e ao padrão de sutura.

O emprego da sutura com ponto simples separado, alternado com ponto de relaxamento permitiu maior expansão dos tecidos e proporcionou melhor drenagem de líquido subcutâneo. Considerações similares foram salientadas por Lazzeri (1994). Paulo et al. (2005) relataram que além dessas particularidades, o padrão de sutura ponto simples separado, alternado com ponto de relaxamento induz, comprovadamente, a menor tensão, pois o compo-

Tabela 2. Resultados da comparação entre o tipo de fio e padrão de sutura empregada no tratamento cirúrgico de hérnia umbilical em bovinos jovens, em propriedades rurais do Estado de Goiás, Distrito Federal e Minas Gerais, entre 1989 e 2009

\begin{tabular}{|c|c|c|c|c|c|}
\hline \multirow{2}{*}{ Grupo } & \multicolumn{2}{|c|}{ Recuperado } & \multicolumn{2}{|c|}{ Não Recuperado } & \multirow{2}{*}{$\mathbf{p}$} \\
\hline & $\mathbf{N}$ & $\%$ & $\mathbf{N}$ & $\%$ & \\
\hline \multicolumn{6}{|l|}{ Algodão } \\
\hline Jaquetão Convencional $^{\mathrm{ab}}$ & 12 & 92,3 & 1 & 7,7 & \\
\hline Jaquetão Modificado $^{\mathrm{a}}$ & 9 & 69,2 & 4 & 30,8 & 0,052 \\
\hline Ponto Simples Separado & 13 & 100 & 0 & 0 & \\
\hline Total & 34 & 87,18 & 5 & 12,82 & \\
\hline \multicolumn{6}{|l|}{ Náilon } \\
\hline Jaquetão Convencional $^{\mathrm{a}}$ & 9 & 69,2 & 4 & 30,8 & \\
\hline Jaquetão Modificado a & 8 & 61,5 & 5 & 38,5 & 0,697 \\
\hline Ponto Simples Separado & 10 & 76,9 & 3 & 23,1 & \\
\hline Total & 27 & 69,23 & 12 & 30,73 & \\
\hline$\overline{\text { Algodão }^{\mathrm{a}}}$ & 34 & 87,17 & 5 & 12,82 & 0,055 \\
\hline Náilon ${ }^{\mathrm{a}}$ & 27 & 69,23 & 12 & 30,76 & \\
\hline
\end{tabular}

Teste: Qui-quadrado. Letras iguais na coluna, para o mesmo tratamento, não diferem estatisticamente $(\mathrm{p}>0,05)$. 
nente longe atua como redutor de tensão, ao passo que o perto, faz o contrário. Assim, diante dos resultados obtidos neste estudo, é possível argumentar que, dependendo do padrão de sutura empregado, a tração excessiva nos pontos de sutura promove isquemia tecidual, sendo o principal fator para a ineficácia da sutura.

Em relação à sutura de jaquetão convencional, destaca-se que essa modalidade de sutura permitiu sobreposição das bordas teciduais e adequada cicatrização. Entretanto, quando o anel herniário for amplo, além da dificuldade de se proceder à sutura e de não conferir aparência cosmética, parece aumentar o índice de recidivas. Conforme mencionado por Lazzeri (1994), a sutura em jaquetão tem indicação no fechamento da parede abdominal, especialmente nas eviscerações, eventrações e na redução de anel herniário, pois confere melhor cicatrização e previne recidiva. Gianlupi \& Trindade (2004) descreveram que, na correção de defeitos da parede abdominal, o padrão de sutura, associado ao tipo de fio apropriado, são os principais fatores que determinam a eficácia do procedimento cirúrgico. Para estes pesquisadores, o objetivo é restaurar a integridade, resistência e o contorno da parede abdominal. Acrescente-se que o material usado para a correção deve ser resistente o suficiente para evitar recidiva. Na Tabela 4, apresentam-se os resultados do tratamento, em relação ao grupo a que os animais pertenciam.

Considerando-se os índices de recuperação dos animais pertencentes a cada grupo, verificou-se que houve cura completa de $12(92,3 \%)$ bovinos pertencentes ao grupo GI, nove $(69,2 \%)$ do GII, $13(100 \%)$ do GIII, nove $(69,2 \%)$ do GIV, oito $(61,5 \%)$ do GV e de dez $(76,9 \%)$ do GVI, conforme observado na Tabela 3. Comparando-se, entre eles, todos os protocolos avaliados, observou-se diferença significativa, quando se confrontaram os resultados obtidos para o GII com os do GIII ( $\mathrm{p}=0,030)$; os do GIII com os do GIV ( $p=0,030)$ e os do GIII com os do GV ( $p=0,013)$.
Porém, comparando-se os outros grupos entre si, não se constatou diferença estatística $(\mathrm{p}>0,05)$. Esses dados mostram que GIII apresentou maior número de animais recuperados. Informações obtidas, comparando-se os tipos de fios, associados ao padrão de sutura e a possíveis particularidades, não foram mencionadas na literatura consultada.

O efeito positivo do tratamento cirúrgico empregado no grupo GIII, utilizando-se o fio de algodão, associado ao ponto simples separado, alternado com ponto de relaxamento, ficou evidente, ao se analisar a evolução clínica do processo, uma vez que todos os $13(100 \%)$ animais desse grupo apresentaram recuperação completa e não apresentaram recidiva. Os resultados alcançados com o tratamento empregado no grupo GI também indicam certa superioridade, em relação aos dos demais tratamentos. Portanto, analisando-se todos esses achados, é possível inferir que a técnica cirúrgica com o fio de algodão associado com a sutura padrão jaquetão convencional, empregada nos animais do GI, eventualmente, também pode ser indicada para essa finalidade e, possivelmente, ao se compará-la com o padrão de sutura em pontos simples, alternando com pontos de relaxamento, empregando-se um número maior de animais, os resultados podem reforçar essa expecativa. Mas, numa análise apenas dos resultados alcançados para o grupo GIII, verifica-se que eles estão de acordo com os de Andrews et al. (2008), os quais citaram que a herniorrafia é perfeitamente corrigida com fio multifilamentar em ponto simples separado.

Dos 78 animais submetidos à intervenção cirúrgica, observou-se, ao decorrer do experimento, deiscência de sutura em $15(19,23 \%)$, sendo quatro (10,2\%) animais em que se empregou o fio de algodão e $11(28,94 \%)$ nos quais se utilizou fio de náilon (Tabela 5). Ocorrência semelhante foi constatada por Sutradhar et al. (2009), ao utilizarem categute cromado na redução do anel herniário. Os auto-

Tabela 3. Resultados do tratamento cirúrgico de hérnia umbilical, comparando os padrões de sutura em relação aos fios, em propriedades rurais do Estado de Goiás, Distrito Federal e Minas Gerais, entre 1989 e 2009

\begin{tabular}{|c|c|c|c|c|c|c|}
\hline \multirow{2}{*}{ Variáveis } & & \multicolumn{2}{|c|}{ Recuperado } & \multicolumn{2}{|c|}{ Não recuperado } & \multirow{2}{*}{$\mathbf{p}$} \\
\hline & & $\mathbf{N}$ & $\%$ & $\mathbf{N}$ & $\%$ & \\
\hline \multirow{2}{*}{ Jaquetão Convencional } & Algodão $^{\mathrm{a}}$ & 12 & 92,3 & 1 & 7,7 & \multirow[t]{2}{*}{0,136} \\
\hline & Náilon $^{\mathrm{a}}$ & 9 & 69,2 & 4 & 30,8 & \\
\hline \multirow{2}{*}{ Jaquetão Modificado } & Algodão $^{\mathrm{a}}$ & 9 & 69,2 & 4 & 30,8 & \multirow[t]{2}{*}{0,680} \\
\hline & Náilon ${ }^{a}$ & 8 & 61,5 & 5 & 38,5 & \\
\hline \multirow[t]{2}{*}{$\overline{\mathrm{PSS}}$} & Algodão ${ }^{\mathrm{a}}$ & 13 & 100 & 0 & - & \multirow{2}{*}{0,066} \\
\hline & Náilon $^{\mathrm{a}}$ & 10 & 76,9 & 3 & 23,1 & \\
\hline Jaquetão Convencional $^{\text {ab }}$ & & 21 & 80,76 & 5 & 19,23 & \multirow{3}{*}{0,123} \\
\hline Jaquetão Modificado ${ }^{\mathrm{a}}$ & & 17 & 65,38 & 9 & 34,61 & \\
\hline Ponto simples separado & & 23 & 88,46 & 3 & 11,53 & \\
\hline
\end{tabular}

PSS - Ponto separado simples.

Teste: Qui-quadrado e Exato de Fisher. Letras diferentes na coluna, para o mesmo tratamento, diferem estatisticamente (p < 0,05).

Rev. Ceres, Viçosa, v. 59, n.1, p. 39-47, jan/fev, 2012 
res relataram que, após duas semanas do procedimento cirúrgico, 21,4\% dos bezerros apresentaram recidivas, bem como enfraquecimento da musculatura no local da cirurgia. Dentre as possíveis causas de recidiva, destacam-se a pressão das vísceras sobre a sutura, o diâmetro do anel herniário, a presença de processo inflamatório e a fragilidade dos tecidos, afirmações que estão de acordo com Nguhiu Mwangi et al. (1991). Para Gonzalo (1994), o excesso de líquido nos espaços intersticiais, associado à formação de vesículas contendo fluido seroso ou serohemorrágico, pode ocasionar espessamento da região do anel herniário, dificultando a reparação tecidual. Rabelo et al. (2005), embasados em estudos experimentais, afirmaram que a retirada excessiva de pele, durante a confecção da incisão elíptica, promove tensão em excesso na ferida cirúrgica, com posterior deiscência da ferida.

Em relação a outras complicações pós-operatórias, constataram-se $52(48,15 \%)$ casos de edema, com ocorrência em todos os grupos, com diferença estatística entre as demais alterações ( $\mathrm{p}<0,05)$. O problema pode estar relacionado com a grande manipulação dos tecidos na região, reação inflamatória acentuada e ao veículo do próprio anestésico local. Achados similares foram menciona- dos por Rabelo et al. (2005), os quais descreveram o edema como principal complicação observada nos animais submetidos à correção de hérnia umbilical, utilizando-se centro tendíneo diafragmático. Essa ocorrência também foi observada por Sutradhar et al. (2009), mas os autores não fizeram qualquer elucidação sobre as possíveis causas.

As demais complicações encontradas foram as fibroses, em 19 (17,6\%) animais; deiscência de ferida, em dez $(9,3 \%)$; casos de miíases, em seis $(5,6 \%)$ e abscessos, em quatro $(3,7 \%)$. Houve também um $(0,9 \%)$ caso de evisceração e um $(0,9 \%)$ óbito. Sutradhar et al. (2009) relataram tais intercorrências na correção de hérnia em bezerros, sem, contudo, informar a frequência. Os autores também advertiram sobre a presença de fístula no abomaso, no local da cirurgia. Nos animais aqui avaliados, tais complicações podem estar diretamente associadas às condições precárias de manejo e aos locais onde os animais foram submetidos aos procedimentos cirúrgicos, situação que pode ter comprometido o controle das infecções no trans e no pós-operatório. Entre outras falhas, destacam-se o excesso de alimentação durante a fase de recuperação, a falta de continuidade da antibioticoterapia prescrita, a manutenção dos animais em locais com excesso de

Tabela 4. Resultados do tratamento cirúrgico de hérnia umbilical em bovinos jovens de acordo com os grupos, em propriedades rurais do Estado de Goiás, Distrito Federal e Minas Gerais, entre 1989 e 2009

\begin{tabular}{|c|c|c|c|c|c|}
\hline \multirow{2}{*}{ Grupo } & \multicolumn{2}{|c|}{ Recuperado } & \multicolumn{2}{|c|}{ Não recuperado } & \multirow{2}{*}{$\mathbf{p}$} \\
\hline & $\mathbf{N}$ & $\%$ & $\mathbf{N}$ & $\%$ & \\
\hline$\overline{\mathrm{GI}^{\mathrm{ABC}}}$ & $12^{\mathrm{a}}$ & 92,3 & $1^{\mathrm{b}}$ & 7,7 & 0,002 \\
\hline GII ${ }^{\mathrm{AC}}$ & $9^{\mathrm{a}}$ & 69,2 & $4^{\mathrm{a}}$ & 30,8 & 0,165 \\
\hline $\mathrm{GIII}^{\mathrm{AB}}$ & 13 & 100 & 0 & 0 & - \\
\hline GIV $^{A C}$ & $9^{a}$ & 69,2 & $4^{\mathrm{a}}$ & 30,8 & 0,165 \\
\hline $\mathrm{GV}^{\mathrm{AC}}$ & $8^{\mathrm{a}}$ & 61,5 & $5^{\mathrm{a}}$ & 38,5 & 0,405 \\
\hline $\mathrm{GVI}^{\mathrm{ABC}}$ & $10^{\mathrm{a}}$ & 76,9 & $3^{\mathrm{a}}$ & 23,1 & 0,052 \\
\hline Total & 61 & 78,20 & 17 & 21,79 & \\
\hline
\end{tabular}

Teste: Qui-quadrado: ${ }^{2} L e t r a s$ iguais na linha, para o mesmo tratamento, não diferem estatisticamente (p > 0,05). ${ }^{\mathrm{A}}$ Letras maiúsculas iguais na coluna, entre os grupos, não diferem estatisticamente (p > 0,05). Grupos I, II e III: Fio de Algodão; Grupos IV, V e VI: Fio de Náilon; Grupos I e IV: Jaquetão convencional; Grupos II e V: Jaquetão modificado; Grupos III e VI: Ponto separado simples com ponto de relaxamento.

Tabela 5. Complicações pós-operatórias de hérnia umbilical em bovinos jovens, em propriedades rurais dos Estados de Goiás, Minas Gerais e Distrito Federal, entre 1999 e 2009

\begin{tabular}{lccccccccc}
\hline \multirow{2}{*}{ Grupo } & \multicolumn{7}{c}{ Complicações } & Total (\%) \\
\cline { 2 - 8 } & Ede & D. fer & Mií & Abs & Fib & Rec & Evi & Óbi \\
\hline I & 9 & 3 & 3 & 1 & 4 & 1 & - & - & $19,44^{\mathrm{A}}$ \\
II & 8 & 2 & 1 & 2 & 3 & 3 & - & 1 & $18,52^{\mathrm{A}}$ \\
III & 11 & 2 & 0 & - & 5 & - & - & - & $16,67^{\mathrm{A}}$ \\
IV & 7 & 1 & 1 & - & 2 & 4 & - & - & $13,89^{\mathrm{A}}$ \\
V & 7 & - & - & 1 & 2 & 4 & 1 & - & $13,89^{\mathrm{A}}$ \\
VI & 10 & 2 & 1 & - & 3 & 3 & - & - & $17,59^{\mathrm{A}}$ \\
\hline Total (\%) & $48,15^{\mathrm{a}}$ & $9,26^{\mathrm{bc}}$ & $5,56^{\mathrm{c}}$ & $3,70^{\mathrm{c}}$ & $17,59^{\mathrm{b}}$ & $13,89^{\mathrm{b}}$ & $0,93^{\mathrm{d}}$ & $0,93^{\mathrm{d}}$
\end{tabular}

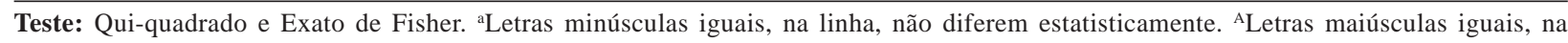
coluna, não diferem estatisticamente (p > 0,05). Ede: Edema; D.fer: Deiscência de ferida; Mií: Miíases; Abs: Abscesso; Fib: Fibrose; Rec: Recidiva; Evi: Evisceração; Óbi: Óbito 
sujidades, as falhas na realização dos curativos diários e o desinteresse da mão de obra auxiliar em seguir as recomendações prescritas. Essa observação encontra respaldo nas informações de Silva et al. (2003), os quais, afirmaram que o sucesso da hernioplastia depende do pré e do pós-operatórios adequados.

Dentre os 16 animais não recuperados, durante a reintervenção, ou por ocasião da necrópsia do animal que veio a óbito, em $11(68,7 \%)$ foram constatadas aderências de estruturas, como omento, abomaso e alças intestinais. Segundo Silva et al. (2003) e Paulo et al. (2005), as aderências, ocorrem após manipulações da parede abdominal, que podem ocasionar isquemia, que constitui numa sequela importante da cirurgia abdominal. Essa alteração ocorre por causa da agressão aos tecidos por parte dos pontos de sutura, originando uma reação inflamatória local e, consequentemente, peritonite, podendo, inclusive, resultar em óbito.

Analisando-se apenas o material de sutura, verificouse que o emprego do fio de algodão resultou em $59(54,6 \%)$ complicações e o do fio de náilon em 49 (45,4\%). Apesar de o fio de algodão desencadear maior número de complicações pós-operatórias, o número de animais com recidivas foi menor, possivelmente, por desencadear maior fibrose local, estando estes resultados de acordo com os achados de Silva et al. (2006). Silva et al. (2003) avaliaram 32 bovinos, machos e fêmeas, com idade entre quatro e 12 meses, com hérnia umbilical recidiva e constataram que, do total de bovinos operados, 27 apresentavam resquícios dos fios de algodão utilizados na laparorrafia precedente, estando muitos deles envoltos por pequenos abscessos, provavelmente resultantes de contaminação do fio. Segundo Lazzeri (1994) e Fossum (2008), a capilaridade do fio de algodão permite abrigar bactérias, predispondo a infecções. Em contrapartida, esse fio tem alta resistência, suporta grandes tensões e permite a confecção de nós seguros. Todavia, podem ocorrer, com seu uso, reações inflamatórias com formação de fístulas e granulomas, o que exige intervenção cirúrgica para sua retirada.

Avaliando o número de complicações em relação com o padrão de sutura sem considerar o fio empregado, verifica-se que os padrões jaquetão convencional, jaquetão modificado e ponto separado, alternado com pontos de relaxamento relacionaram-se, respectivamente, com 36 (33,3\%), $35(32,4 \%)$ e 37 (34,3\%) alterações, durante o pósoperatório. Todavia, com o padrão de sutura em pontos separados ocorreram apenas três $(18,7 \%)$ recidivas, de um total de 16 casos, envolvendo recidiva e evisceração. Neste contexto, ressaltam-se as afirmações de Lazzeri (1994), o qual relatou que o ponto simples separado, alternado com pontos de relaxamento diminui a possibilidade de isquemia e de deiscência da ferida, por proporcionar maior expansão dos tecidos.
Ponderando-se sobre os casos de recidivas em relação ao padrão de sutura, em todos os grupos, verifica-se que o emprego do jaquetão resultou em cinco $(31,25 \%)$ casos de insucesso e o do jaquetão modificado em nove $(56,25 \%)$, incluindo uma evisceração $(6,25 \%)$ e um óbito $(6,25 \%)$ dentre os 16 bovinos. Esses achados não condizem com os obtidos por Silva et al. (1999), os quais observaram que a sutura em padrão jaquetão com invaginação das aponeuroses abdominais proporcionou adequada resistência e menor incidência de recidivas, quando comparada com a sutura jaquetão convencional. A parede abdominal é uma estrutura importante para as funções respiratória e digestiva, pois previne eventração visceral, fornece suporte muscular dinâmico, promove a aproximação livre de tensão e assegura que a parede reconstruída tenha sua força aumentada ao longo do tempo, conforme afirmaram Paulo et al. (2005).

\section{CONCLUSÕES}

A ocorrência de hérnia umbilical em bovinos é expressiva e a herniorrafia, empregando-se fio de algodão em padrão de pontos sutura simples separados, alternando com pontos de relaxamento apresenta mais complicações pós-operatórias, mas recupera maior número de animais. O fio e padrão de sutura, o manejo pós-operatório, a resposta individual e o tamanho do anel herniário influenciam na reabilitação dos animais e na redução das complicações pós-operatórias.

\section{REFERÊNCIAS}

Andrews AH, Blowey RW, Boyd H \& Eddy ERG (2008) Medicina bovina: doenças e criação de bovinos. $2^{\mathrm{a}}$ ed. São Paulo, Roca. $1080 \mathrm{p}$.

Bahr C \& Distl O (2005). Frequency of congenital anomalies in cattle: results from the practice in comparison with literature. Dtsch Tierarztl Wochenschr, 112:149-154.

Baird AN (2008) Umbilical in Calves. Veterinary Clinics of North America: Food Animal Practice, 24:467-477.

Citek J, Øehout V \& Hájková J (2009) Congenital disorders in the cattle population of the Czech Republic. Czech Journal of Animal Science, 54:55-64.

Cuffari L (1997) Considerações gerais em odontologia dos fios de sutura. Jornal Brasileiro de Odontontologia Clínica, 1:43-47.

Dias Filho FC, Silva LAF \& Silva OC (2001) Relatos de experiências de campo. In: Silva LAF, Fioravanti MCS, Dias Filho FC \& Eurides D (Eds) Sanidade dos bezerros leiteiros: da concepção ao desmame. Goiânia, Talento Gráfica e Editora. p.80-87.

Erdoðan HM, Unver A, Çitil M, Günes V, Arslan MO, Tuzcu M \& Gökçe HI (2009) Dairy farming in the district of Kars, Turkey: III. Health Neonatal calf. Turkish Journal of Veterinary and Animal Sciences, 33:185-192.

Figueirêdo LJC (1999) Onfalopatias de bezerros. $1^{\text {a }}$ ed. Salvador, EDUFBA. 95p.

Fossum (2008) Cirurgia de Pequenos Animais. $3^{\text {a }}$ ed. São Paulo, Roca. 1313p. 
Gianlupi Á \& Trindade MRM (2004) Comparison between fixation of polypropylene mesh with polypropylene suture and polyglactin 910 suture for treatment of muscle-aponeurotic defects of abdominal wall: experimental study in rats. Acta Cirúrgica Brasileira, 19:94-102.

Gonzalo JMC (1994) Cirurgia Veterinária. 1ª ed. Madrid, Interamericana. $884 \mathrm{p}$.

Herrmann R, Utz J, Rosenberger E, Doll K \& Distl O (2001) Risk factors for congenital umbilical hernia in German fleckvieh. The Veterinary Journal, 162:233-240.

Lazzeri L (1994) Técnica operatória veterinária. $1^{a}$ ed. São Paulo, Manole. 415 p

Nguhiu Mwangi JA, Munyua SJM, Mbithi PFM, Mbiuki SM \& Mogoa EGM (1991) How to improve the prognosis of ventral abdominal hernias in large animals: "modified" overlapping technique. Bulletin of Animal Health Production African, 39:315-320.

Paulo NM, Lima FG, Juarez Júnior TS, Fleury LFF, Sant'Ana FJF, Borges AC \& Telles TC (2005) Seringueira's latex membrane (Hevea brasiliensis) with and without polylysine $0,1 \%$ and marlex mesh for the reconstruction of iatrogenic abdominal wall defects in rats. Acta Cirurgica Brasileira, 20:305-310.

Peiró JR, Lucato B, Mendes LCN, Ciarlini PC, Feitosa FLF, Bonello FL, Maemura SM, Soares GT, Santana AE \& Perri SHV (2009) Evaluation of cytologic and biochemical variables in blood, plasma, and peritoneal fluid from calves before and after umbilical herniorrhaphy. American Journal of Veterinary Research, 70:423-432.

Rabelo RE, Sant'Ana FJF, Paulo NM, Romani AF, Silva LAF, Viu MAO, Alves CB, Lima CRO \& Silva OC (2005) Use of the polilisine $0.1 \%$ and poliamide, latex membrane in the surgical correction of recidivating umbilical hernias in dairy cattle. Acta Scientiae Veterinariae, 33:169-175.

Rings DM (1995) Umbilical hernias, umbilical abscesses, and urachal fistulas. Surgical considerations. Veterinary Clinics of North America: Food Animal Practice, 11:137-148.

Sampaio IBM (2007) Estatística aplicada à experimentação animal. 3.ed. Belo Horizonte, FEPMVZ. 264p.

SAS, Institute Inc. Statistical Analysis System user's guide (2002) Version 9, 13ª ed. São Paulo.
Silva LAF, Fioravanti MCS, Eurides D, Juliano RS, Acypreste CS \& Borges GT (1999) Sobreposição com invaginação das aponeuroses dos músculos abdominais no reparo de hérnias umbilicais em bovinos. Veterinária Notícias, 5:63-67.

Silva LAF, Neto JBP, Chiquetto CE, Fioravanti MCS, Eurides D, Borges GV, Atayde IB \& Rabelo RE (2000) Herniorrafia umbilical em bovinos e avaliação do pós-operatório. Ciência Animal Brasileira (Suplemento). 1:126.

Silva LAF, Fioravanti MCS, Dias Filho FC \& Eurides D (2001) Sanidade dos bezerros leiteiros: da concepção ao desmame. $1^{\text {a }}$ ed. Goiânia, Talento Gráfica e Editora. 87p.

Silva AF, Paula NJB, Eurides D, Chiquetto CE, Fioravanti MCS, Machado GV, Borges NC, Rabelo RE \& Silva CA (2003) Bovine umbilical herniorraphy: Technical analysis and postoperatory evaluation. Arquivo de Ciência Veterinária e Zoologia, 6:105108

Silva LAF, Soares LK, Bernardes KM, Barbosa VT, Lima IR, Esteves GIF, Costa AC, Coelho CMM, Lima CRO, Silva MAM \& Martins LR (2006) Evisceração após herniorrafia umbilical em um bovino: relato de caso. Veterinária Notícias, 12:87.

Sobayil FA \& Ahmed AF (2007) Surgical treatment for different forms of hernias in sheep and goats. Journal of Veterinary Science, 8:185-191.

Steenholdt C \& Hernandez J (2004) Risk factors for umbilical hernia in Holstein heifers during the first two months after birth. Journal of the American Veterinary Medical Association, 224:1487-1490

Steiner A \& Lejeune B (2009) Ultrasonographic Assessment of Umbilical Disorders. Veterinary Clinics of North America - Food Animal Practice, 25:781-794.

Sutradhar BC, Hossain MF, Das BC, Kim G \& Hossain MA (2009) Comparison between open and closed methods of herniorrhaphy in calves affected with umbilical hérnia. The Journal of Veterinary Science, 10:343-347.

Sylvestre A, Wilson J \& Hare J (2002) A comparison of 2 different suture patterns for skin closure of canine ovariohysterectomy. The Canadian Veterinary Journal, 43:699-702.

Turner AS \& McIlwaith CW (2002) Técnicas Cirúrgicas em Animais de Grande Porte. $1^{\text {a }}$ ed. São Paulo, Roca. 341p. 